Journal of Computer Science 3 (7): 540-545, 2007

ISSN 1549-3636

(C) 2007 Science Publications

\title{
A Web-based Interactive Real Laboratory for Process Engineering Education
}

\author{
${ }^{1,2}$ Maher Chaabene, ${ }^{2}$ Kamel Mkaouar and ${ }^{2}$ Mohamed Ouali \\ ${ }^{1}$ The High Institute of Technological Studies (ISET) - Sfax, Tunisia \\ ${ }^{2}$ Unit of Machine Control and Power Grid (CMERP) -ENIS-Tunisia
}

\begin{abstract}
An online supervision and control of a laboratory designed for process engineering education was conceived. The principal aim was to minimise the time access of an operator when manipulating laboratory features. Hence, the Time delay due to Internet traffic was overcome by using a Web Embedded Server (WES). A human-computer interactive interface using JAVA Script was developed so as to control multi-user access. The multi-user management was ensured by means of a PHP-based dynamic pages using sessions' technique, implemented via an APACHE server in the local control system. Approach validation was conducted on a didactic lift considered as an engineering educational process. Online experimentation of the laboratory showed similar behaviour to the local manipulation. Moreover, the access time to laboratory features was significantly reduced and many facilities were offered to students.
\end{abstract}

Key words: Embedded systems, Internet Supervision and control, e-learning, web-server

\section{INTRODUCTION}

These last years, impressive advances in Internet have made this network the most successful. Standards, technologies and Web-implementations do not cease improving and diversifying. Nowadays, the use of a Web navigator for information exchange has become well-known by the large public. The idea consists of exploiting this infrastructure with its standards of communication to supervise and control educational laboratory instruments and features over the Web. Thus, it becomes possible to reach these distant apparatuses by using a standard Web navigator.

Researches focused on developing virtual features for distant learning purposes including Web-based virtual control laboratories ${ }^{[1]}$ and Web-based learning resources libraries $\left.{ }^{[2,} 3\right]$. However, few publications present real Web-control laboratory. The encountered difficulties reside in limitation, caused by Internet environment features, such as the shortage of Webcontrol devices, Internet time delay, multiple users' collaboration and Web-related safety ${ }^{[4]}$.

This paper suggests supervision and control over the Web of a real laboratory so as to teach interactively engineering materials. The approach was based on a Program Logic Controller (PLC) connected to a miniature Web Embedded Server (WES). Since this later was configured to manage only our educational process, the Internet time delay had to be reduced.
Furthermore, multi-user access control was investigated via a Web application.

The various sections are organised as follows. Section 2 fixes requirement specification of the Internet-based laboratory. The online supervision and control approach is investigated in section 3 . Section 4 shows the system implementation. The results' valorisation are given in section 5. Section 6 provides conclusion and perspectives.

\section{REQUIREMENT SPECIFICATION}

The first task to be accomplished when designing a real time control system is specifying the requirements ${ }^{[1]}$. This should determine the right control structure. In our case, the requirements should include process monitoring and control objectives that are entirely achievable through the Internet control level. Thus, the conflicting tradeoffs between goals and constraints of the system have to be identified and resolved.

Requirement analysis: Several specification methods are used in research work: System Analysis Design Technique (SADT), Unified Modelling Language (UML), and Structured Analysis for Real Time (SART), etc. These methods depend on the nature and constraints of the system to develop. As we consider the aimed system has a Web-related traffic delay constraint, we adopt the SART method in order to take into

Corresponding Author: $\quad$ Rte Mahdia Km 2, BP88A, ElBustan 3099- Sfax, Tunisia 
account the real time behaviour. This method consists of three stages. First, a context schema analyses the fundamental and the secondary system tasks to be accomplished and their interactions (fig.1). In a second stage, a preliminary schema gives details for each task so as to define all system components with their interactivity and behaviour (fig.2). Finally the ordering schema (fig. 3) shows all lift orders details. Table 1 gives the data dictionary that contains the whole system data base definition.

\begin{tabular}{ll}
\hline DO1 / DO2 / DO3 / DO4 & Door of the $1^{\text {st }} / 2^{\text {nd }} / 3^{\text {rd }} / 4^{\text {th }}$ floor is opened \\
DC1 / DC2 / DC3 / DC4 & Door of the $1^{\text {st }} / 2^{\text {nd }} / 3^{\text {rd }} / 4^{\text {th }}$ floor is closed \\
GDLF1 / GDLF2 / GDLF3 & Get down the lift to the $1^{\text {st }} / 2^{\text {nd }} / 3^{\text {rd }}$ floor \\
RLF2 / RLF3 / RLF4 & Raise the lift to the $2^{\text {nd }} / 3^{\text {rd }} / 4^{\text {th }}$ floor \\
RF2 / RF3 / RF4 & Order to the lift to raise to the $2^{\text {nd }} / 3^{\text {rd }} / 4^{\text {th }}$ \\
GDF1 / GDF2 / GDF3 & Order to the lift to get down the to the $1^{\text {st }} / 2^{\text {nd }} /$ \\
& $3^{\text {rd }}$ floor \\
SON1 / SON2 / SON3 / SON4 & Switch on the lamp of the $1^{\text {st }} / 2^{\text {nd }} / 3^{\text {rd }} / 4^{\text {th }}$ floor \\
SONL & Switch on the lamp of the lift \\
SOF1 / SOF2 / SOF3 / SOF4 & Switch off the lamp of the $1^{\text {st }} / 2^{\text {nd }} / 3^{\text {rd }} / 4^{\text {th }}$ floor \\
LF1 / LF2 / LF3 / LF4 & Display the state of the lamp of the $1^{\text {st }} / 2^{\text {nd }} / 3^{\text {rd }} / 4^{\text {th }}$ \\
& floor \\
LST & Lift state \\
CYPR & Display the cycle's program \\
Tick & Clock tick \\
& Table 1 : Data dictionary \\
\hline
\end{tabular}

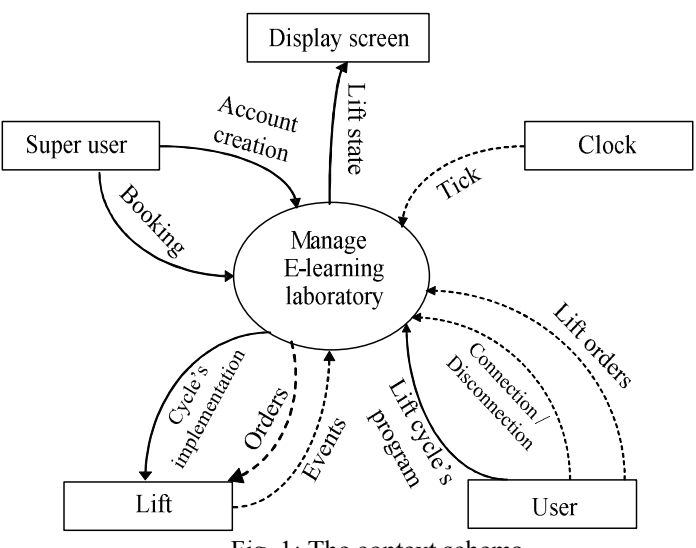

Fig. 1: The context schema

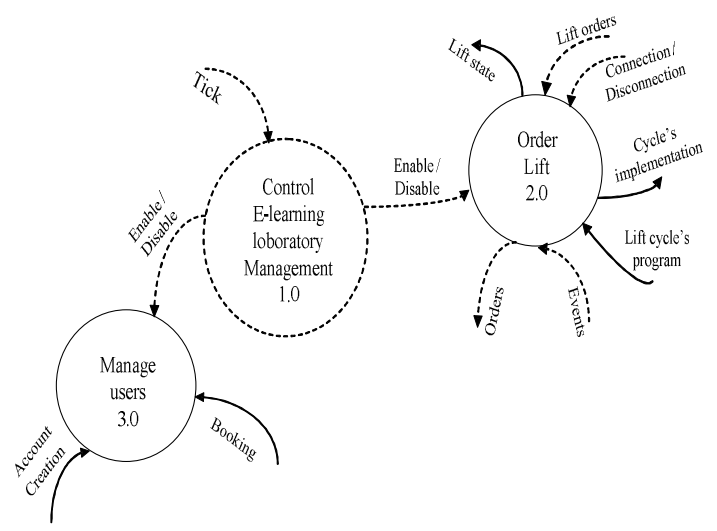

Fig. 2: The preliminary transformation.

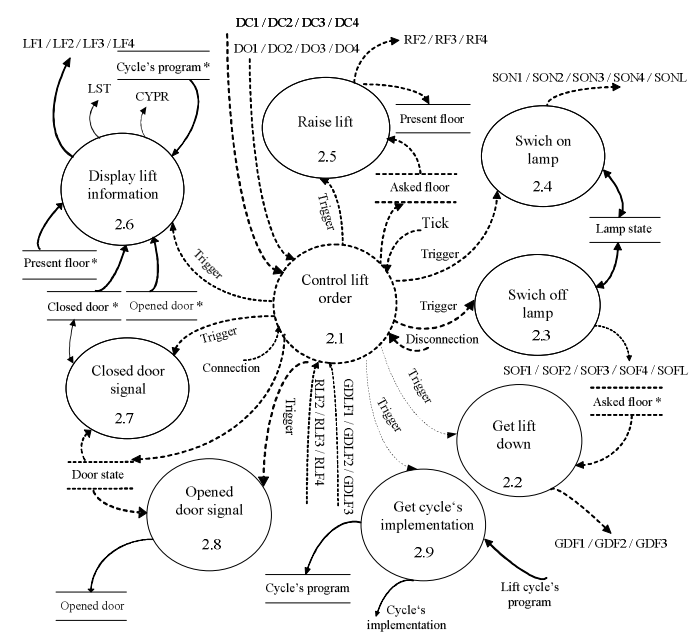

Fig. 3: The Order lift diagram

Discussion on control constraints: The main constraints of a Web-control system are the uncertain Internet time-delay, the multi-user access and conflict resolution and the system safety cover. The multi-user access and the system safety constraints have been largely discussed through bibliography. Multiple solutions have been suggested in order to overcome these constraints. These solutions are essentially based on software applications ensuring users' priority management and system safety checking. As for time delay constraint, it is still under study. In what follows we discuss this constraint then we give our adopted approach.

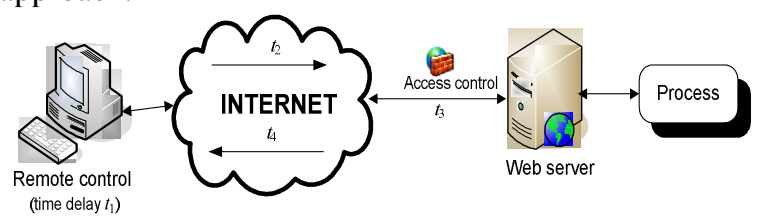

Fig. 4: Web control system constraints

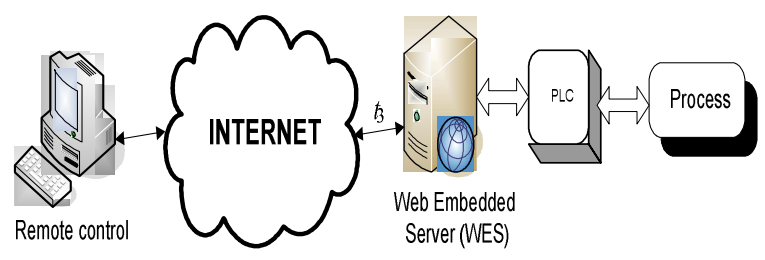

Fig. 5: The process is directly connected to the WES 


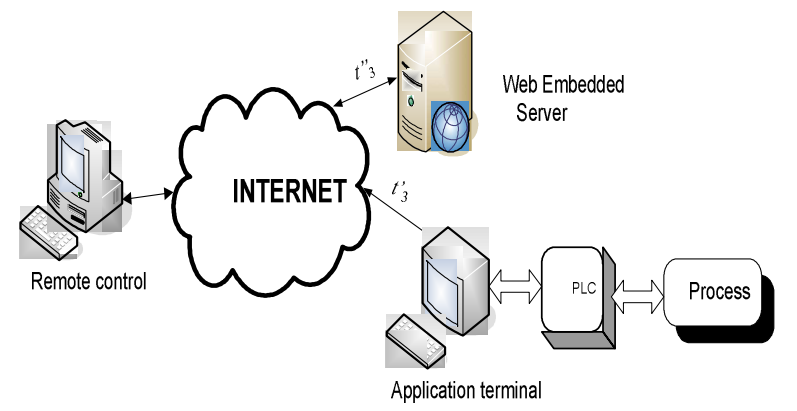

Fig. 6: The process is connected to the WES via an application terminal

The Data flow between Web-based process and Internet clients fixes the efficiency of supervision and control operations. One of the difficulties in Internet-based process control is the dynamic delay caused by the Internet traffic. Cycles' times to a process control operation over the Internet are shown by figure 4 .

The total time needed to perform a Web process control operation $^{[4,7]}$ is: $\mathrm{T}=t_{1}+t_{2}+t_{3}+t_{4}$, where:

$t_{1}$ : time delay in making control decision by a remote operator.

$t_{2}$ : time delay in transmitting a control command from the remote operator to the local system.

$t_{3}$ : execution time of the local system to perform the control action.

$t_{4}$ : time delay in transmitting the information from the local system to the remote operator.

Many approaches have been used in order to minimize the amount $\mathrm{T}$ by reducing its time delay terms $t_{1}, t_{2}, t_{3}$ and $t_{4}$.

First, since the amount $t_{1}$ depends only on the operator reaction, it cannot be minimized.

Second, the Internet time delays $t_{2}$ and $t_{4}$ increase with distance and depend essentially on the number of nodes traversed. The Internet time delays $t_{d}(k)\left(t_{2}\right.$ or $\left.t_{4}\right)$ at instant $k$ can be calculated as follows ${ }^{[6]}$.

$$
t_{d}(k)=\sum_{i=0}^{n}\left[\frac{l_{i}}{C}+t_{i}^{R}+t_{i}^{L}(k)+\frac{M}{b_{i}}\right]=\sum_{i=0}^{n}\left(\frac{l_{i}}{C}+t_{i}^{R}+\frac{M}{b_{i}}\right)+\sum_{i=0}^{n} t_{i}^{L}(k)=d_{N}+d_{L}(k) .
$$

Where $l_{i}$ is the $i$ th length of link, $C$ the speed of light, $t_{i}^{R}$ the routing speed of the $i^{\text {th }}$ node, $t_{i}^{L}(k)$ the delay caused by the $i^{\text {th }}$ node's load, $M$ the amount of data, and $b_{i}$ the bandwidth of the $i^{\text {th }}$ link.

The term $d_{N}$ depends on network performances and can only be minimized by improving the Internet load: the processing speed of nodes, the connection bandwidth type, the transmission speed, etc. As for $d_{L}(k)$, it is a time-dependent term which can be reduced by choosing a control architecture which is insensitive to the time delay.

Finally, reducing the time delay $t_{3}$ requires an increase of the local system speed to perform an action. Thus, the most useful approach consists in using a virtual control strategy. Although it is the best solution since it is not sensitive to the time delay $t_{3}$, this method presents the handicap of virtual applications and cannot convey real information between the remote control operator and the local control system.

\section{ONLINE SUPERVISION AND CONTROL}

Our approach consists in configuring a WebEmbedded Server (WES) connected to a Programmable Logic Controller (PLC). The PLC ensures the process supervision and control. At the same time it communicates with the WES by receiving instructions and sending reports. As for the WES, it communicates through the Web with distant users. This approach must offer a dialogue with a real process. The remote control operator sends his requests (process control) to the WES which answers regularly (process acquisition) thanks to a local program pointing PLC data base.

Two different architectures are often used in process control via Internet ${ }^{[5,8,9]}$.

In the first architecture (figure 5) the process is directly connected to the WES via the PLC. As the process is very close to the WES, this involves a significant decrease of the access time $t_{3}$.

As for the second architecture, (figure 6) the process is connected via an application terminal and a PLC to Internet network. The communication between the WES and the process is guaranteed by the Web. This architecture offers a portable application since the process does not need to be near the WES. In addition, several processes in different places could be managed by the same WES. Yet, a permanent connection between the application terminal and the WES is required. Moreover, the time delay $t_{3}$ increases considerably compared to the first architecture because it becomes equal to $\left(t_{3}{ }_{3}+t{ }_{3}\right)$.

As our principal objective is the minimization of access time $t_{3}$, we have selected the first architecture in order to develop our application.

\section{SYSTEM IMPLEMENTATION}

The WES is an e-Device that permits to manage and control remotely any equipment from a standard 
Internet navigator (Internet Explorer, Netscape, Mozilla, etc.). Thus, an Internet site allows full management of the equipment simply by using a terminal connected to Internet or Ethernet. The WES solution integrates all necessary components to transform remotely a non-communicating standard machine into controllable equipment from a standard Web navigator ${ }^{[10]}$

Equipped with a supervision and control program, the PLC is connected to a four stage mini-lift through its input/output lines. This later is protected against operators' wrong movements by hardware system. The data convey between the PLC and the WES through a serial connection RS232C using MEWTOCOL protocol (protocol of Matsushita). As for the WES, it ensures the communication with users through an Ethernet (10 base T) connection. Figure 7 gives the system implementation details.

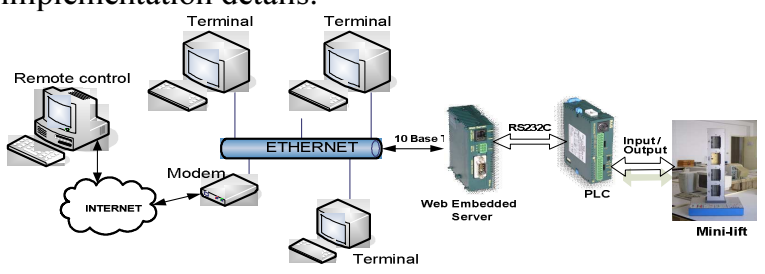

Fig. 7: System implementation

Access control system: The access control system was developed basing on sessions' technique. It supports two types of users:

- Ordinary users who are subscribers having login and password. They are authorised to benefit only from supervision services (visualisation of the lift state).

- A privileged user who is an ordinary unique user possessing access rights that allow him to handle direct orders on the lift as well as to implement a cycle program in PLC. The program commands can be entered in the form of: Ladder, schema of functional context, list of instructions, functional block diagram or structured text. This type of user is identified by an access code available during a fixed time period and obtained after a preliminary booking.

Figure 8 gives flowchart details of the users' access management. As for figure 9 , it shows an illustration of the subscription and the booking Web page.

Web user interface: This phase consists to establish a human-computer interactive Web page in HTML format that ensures the software system configuration (WES and PLC), the communication with users and the system access control. The work validation is conducted on the local network of the high institute of technological studies - Sfax, Tunisia (ISET).
The WES, which is an advanced system, was acquired from NAIS Control Company. Its configuration requires appropriate software called FP Web-Server Configuration: The main menu parameters are fixed so as to minimize the time delay $t_{3}$ and to guarantee a high access control to the process:

- The WES manages only one PLC.

- Information is returned to users by EMAIL.

- The WES access is controlled by password and is reserved to subscribed users.

- The WES accepts three subscribed users at the same time. Only the privileged user is authorized to handle the PLC.

The remote control home page is designed so as to offer maximum flexibility, clarity and effectiveness to the user. It puts forward three control icons: supervision, control orders and cycle programming. Nevertheless, some rights are reserved for safety checking. Page animation uses many multimedia tools that provide information in textual, vocal and graphic forms.

An APACHE server is installed to provide, through supervision icons, home page images and all information about lift state: stage number, emergency key, alarm, door sensor, etc. In addition, control icons allows access, via PLC, to lift straightforward commands such as light switching of the lift box, alarm sound and stage reaching. Finally, cycle programming icons allows conception, adjustment, implementation and testing of an operating cycle algorithm by means of FP Win Pro software.

\section{RESULTS' VALORISATION}

The current prototype system is implemented at the High Institute of Technological Studies (ISET) of Sfax which counts about 3000 students since 2005. More than $30 \%$ of them are asked to handle the same work on the mini-lift. In the traditional teaching method, each student first elaborates a flowchart allowing the minilift function in different control modes by applying standard methods and tools (GRAFCET, Petri Network, etc.). Second, the established diagram must be programmed on a micro-computer connected to the mini-lift through the serial port, then tested by verifying the cycle's stages. This lab course is included in the studying plan of all profiles as process control and automation materials. The teaching method has involved time access barrier when using the system (mini-lift) and a non homogenous student's knowledge evaluation.

Since our main objective is the technological improvement of the lab control, we have chosen to apply the habitual learning content and evaluation methodology which presented no failure. The effectiveness of the proposed system appears essentially in the optimal time sharing of the system, the time free 


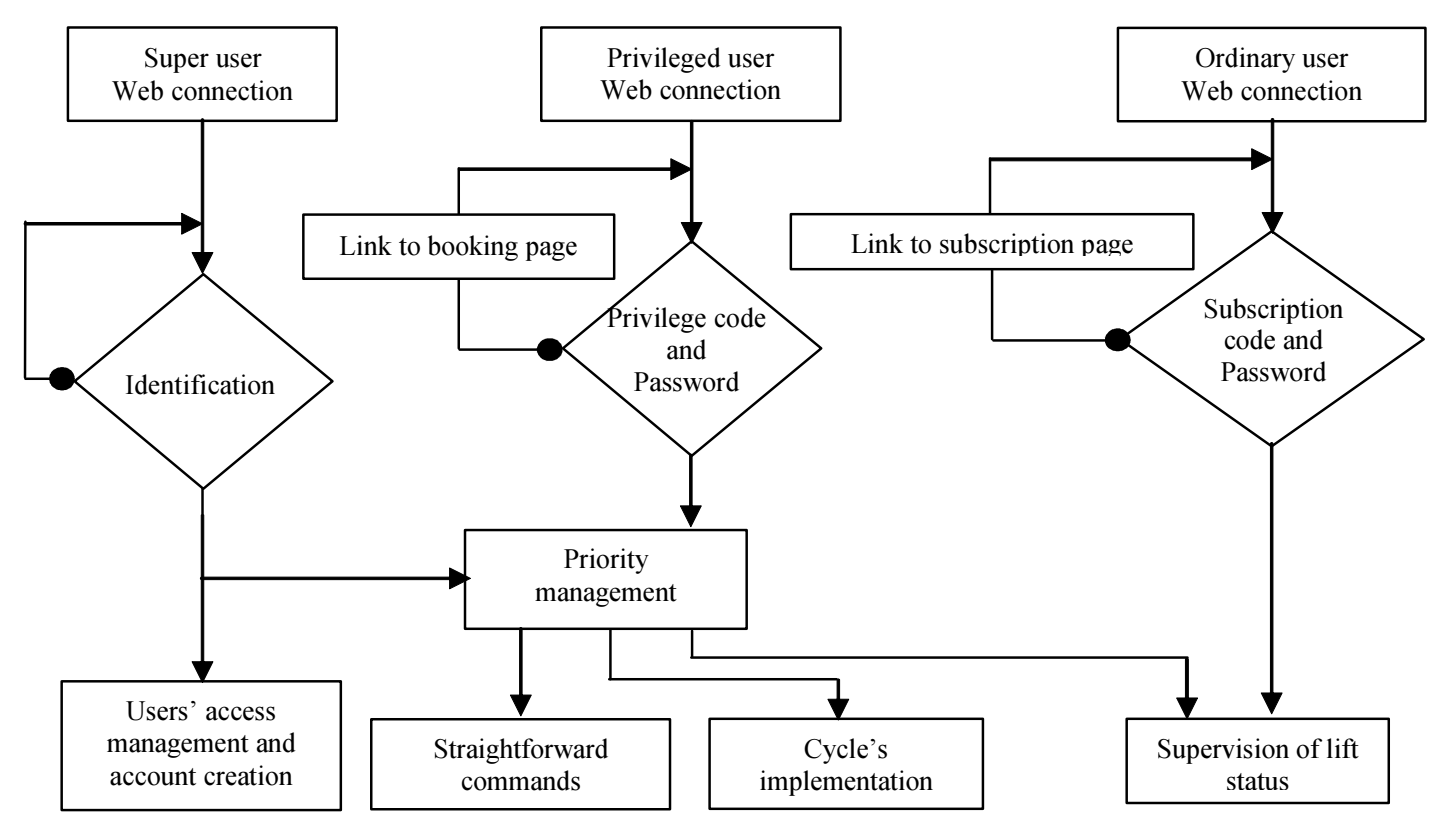

Fig. 8: flowchart details of users' access.

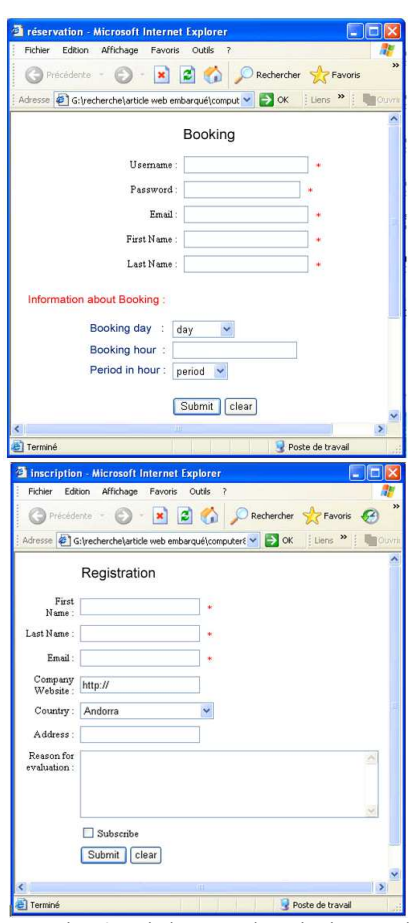

Fig. 9: Links to subscription and booking.

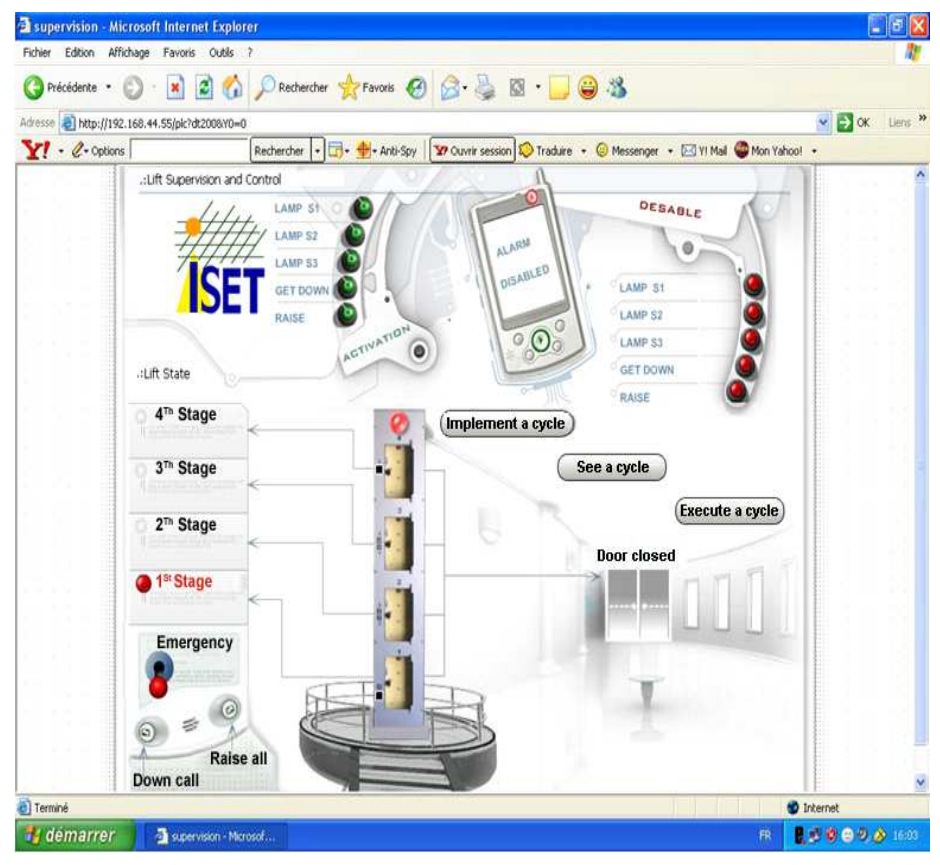

Fig. 10: Remote control home page 
given to students to handle the work and the judicious evaluation of students' applications.

By adopting the new teaching method, many facilities and favours have been offered to the student:

- Since it deals only with the network management and a limited number of users, the WES performs a much reduced task compared with that of usual servers. As for the PLC, it is completely dedicated to the process supervision and control. Consequently, this strategy generated a remarkable reduction in access time $\left(t_{3}\right)$ to the process (\$2.2). Nevertheless this architecture performance depends on the WES because this later has to manage the network communication as well as the dialogue with PLC. The approach assumes, also, the use of powerful transmission support.

- The remote control is accomplished by using the Servlet and Socket concepts (network library allowing interface between the programs and the network layer) available in the JAVA Script programming language. Since it is an object oriented programming, this language offers several functionalities so as to generate applications of great flexibility.

- Browser applets provide interactive demonstrations on the operation manner of the system.

- Preliminary tasks (system study, simulation, functioning diagram implementation, etc.) are developed online without system occupation.

- Students' access to the system for testing their work during all times after booking.

- Work evaluation is ensured online by a commission of experts and returned to students by E-mail.

\section{CONCLUSION}

The supervision and control of a Web-based real laboratory is proposed. In order to minimise the process access time obstacle, a Web Embedded Server is installed and connected to a PLC using real time architecture. A Web interactive page is implemented offering a satisfactory access control and a users' conflict resolution. Two categories of users (privileged and ordinary) have appropriate access rights. The work was carried out on a didactic lift and is being extended in the aim to connect other features to the conceived lab.

\section{REFERENCES}

1. Dongil S., Yoon E. S., Kyung Y. L., Euy S. L. 2002, A web-based interactive virtual laboratory system for unit operations and process systems engineering education: issues, design and implementation. Computers \& Chemical Engineering, 26, 319-330

2. Hough M., Marlin T. 2000, Web-based interactive learning modules for process control. Computers and Chemical Engineering,24,1485 - 1490

3. Chiaming Y. Wu-Jeng L. . 2003, Web-based learning and instruction support system for pneumatics. Computers \& Education Volume 41, Issue 2, Pages 107-120

4. Yang S.H., Chen X., Alty J.L. 2003, Design issues and implementation of internet-based process control systems. Control engineering practice, 11, 709-720

5. Ko C. C., Chen B. M., Chen J., Zhuang, Y., Tan K. C. 2001, Development of a web-based laboratory for control experiments on a coupled tank apparatus. IEEE Transactions on Education, 44(1),76-8

6. Han K. H., Kim S., Kim Y. J., Kim J. H. 2001, Internet control architecture for Internet-based personal robot. Autonomous Robots, 10, 135-147

7. Luo R.C., Chen T.M. 2000, Development of a multi-behaviour based mobile robot for remote supervisory control through the Internet. IEEE Transaction on Software Engineering, 20(9), 684707

8. Mahalik N.P., Ryu J., Ahn B.H. 2005, Simulation integrated management layer for real-time embedded DCN. Computer standards and interfaces (Article in press)

9. Shengwei W., Junlong X. 2002, Integrating building management system and facilities management on the Internet. Automation in construction, 11, 707-715

10. Yang S.H., Chen X., Alty J.L. 2002, Development of a distributed simulator for control experiments through the Internet. Future Generation Computer Systems, 18(5), 595-611. 